\title{
Study of Low Temperature Magnetic Properties of a Single Chain Magnet With Alternate Isotropic and Non-Collinear Anisotropic Units
}

\author{
Shaon Sahoo, Jean-Pascal Sutter and S. Ramasesha
}

\begin{abstract}
Here we study thermodynamic properties of an important class of single-chain magnets (SCMs), where alternate units are isotropic and anisotropic with anisotropy axes being noncollinear. This class of SCMs shows slow relaxation at low temperatures which results from the interplay of two different relaxation mechanisms, namely dynamical and thermal. Here anisotropy is assumed to be large and negative, as a result, anisotropic units behave like canted spins at low temperatures; but even then simple Ising-type model does not capture the essential physics of the system due to quantum mechanical nature of the isotropic units. We here show how statistical behavior of this class of SCMs can be studied using a transfer matrix (TM) method. We also, for the first time, discuss in detail how weak inter-chain interactions can be treated by a TM method. The finite size effect is also discussed which becomes important for low temperature dynamics. At the end of this paper, we apply this technique to study a real helical chain magnet.
\end{abstract}

Keywords Single chain magnets ·Alternate isotropic and anisotropic units · Inter-chain interaction

\section{Introduction}

In the area of magnetism, the field of single chain magnets (SCMs) has attracted huge interest due to its potential use in dense data storage. Magnetism in one dimension is not only interesting from academic point of view, it has also created wide interest for application in technology. A huge body of research has already gone into understanding the physics of SCMs with the aim of designing systems with desired properties [1, 2, 3, 4, 5].

Though it is theoretically impossible to have a spontaneous magnetization at finite temperature in strictly one dimension, apparently there is no upper limit for the relaxation time of induced magnetization. In the field of SCMs, the focus therefore is to increase the relaxation

\section{S. Sahoo}

Department of Physics, Indian Institute of Science, Bangalore 560012, India

e-mail: shaon@physics.iisc.ernet.in

J.-P. Sutter

Laboratoire de Chimie de Coordination, Université de Toulouse, F-31077 Toulouse, France

e-mail: sutter@lcc-toulouse.fr

S. Ramasesha

Solid State \& Structural Chemistry Unit, Indian Institute of Science, Bangalore 560012, India

e-mail: ramasesh@sscu.iisc.ernet.in 
time by combining two modes of relaxations, namely, dynamic relaxation (creation and movement of domain wall(s) along chain) and thermal relaxation (hopping across anisotropy barrier). This is expected to be achieved by synthesizing a spin chain with anisotropic units. While synthetic chemists are exploring different chemical possibilities, theoreticians are developing new techniques to study these compounds. The theoretical studies in return help to understand the paradigms involved in increasing the relaxation time.

Modeling a SCM has to take into account the chemical and geometrical structure of chain units that the system possesses [6, 7]. With quantum units, exact numerical diagonalization technique is not feasible, due to large size of the system, particularly so at low temperatures as correlation length grows exponentially as the temperature is lowered and the system size to be studied should at least have dimension of the correlation length. While a large number of SCMs have been synthesized over the years, here we will study an important class of SCMs in detail. In this class of SCMs, units are alternately isotropic and anisotropic. Furthermore, anisotropy axes are not collinear and since anisotropy is large and negative, the anisotropic units can be thought of as canted Ising spins at low temperatures. It is worth noting that, in this situation had the anisotropy axes been collinear, we could have used a simple one dimensional Ising model as only one component of the spins of the isotropic sites would be coupled to the anisotropic units. But the class of SCMs we wish to model, has non-collinearity of the anisotropy axes, therefore different components of the spin of the isotropic sites would be involved at different sites; as a result, we have to treat the isotropic spins quantum mechanically. Here we note that though all the interactions are quantum mechanical in nature, some of them become insignificant at low temperatures due to highly preferred spin-direction of some sites. Herein, we model these type of systems exactly by transfer matrix method and study their low temperature statistical properties. We also discuss the issue of interchain interactions explicitly, which has been a long pending issue in this field [2].

There are some related theoretical/numerical studies which deserve a brief mention here. Alternative classical-quantum (or anisotropic-isotropic) spin systems have been studied previously [8, 9, 10, 11, 12, 13, 14]. There, classical nature of the spins is assumed from correspondence principle, due to large value of the spin $\mathrm{S}$, of the quantum spins. In an another work, a helical spin chain with alternately classical spin (Ising-type) and quantum spin (coming from organic radical) spins is studied [15]. But here the quantum spins are reduced to classical spins by approximating Ising direction by averaging directions of their neighboring classical spins.

Before we present our method in Sec. 3, we present in the next section (Sec. 2) a brief discussion of a real system which can be modeled to be in the class of SCMs we are interested here. This real example should clarify the present modeling issues and provide the motivation for the model studied in this paper. In Sec. 4, we apply our technique to the real system and present our results. We conclude our work in Sec. 5. 


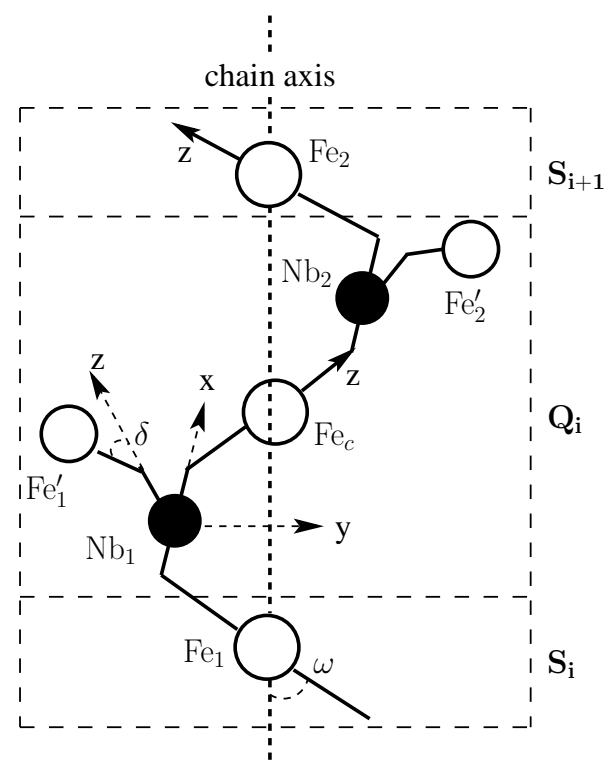

Figure 1: One structural unit of $\mathrm{Fe}_{2} \mathrm{Nb}$ chain is shown here. $\omega$ is the angle made by anisotropy axis of in-chain $\mathrm{Fe}$-ions with chain-axis. $\delta$ is the angle made by anisotropy axis of off-chain $\mathrm{Fe}$ ion with $\mathrm{z}$-axis of associated $\mathrm{Nb}$-ion. $\alpha$ is the angle made by local $x y$-plane of $\mathrm{Nb}$ with the plane formed by all alternate in-chain Fe-ions (here $\mathrm{Fe}_{1}$ and $\mathrm{Fe}_{2}$ ) along with their anisotropy axes. Alternate classical and quantum units are also indicated here by dotted boxes (see Sec. 3).

\section{Description of a Real System}

Recently a compound, we refer to as $\mathrm{Fe}_{2} \mathrm{Nb}$, whose chemical formula is $\left[\left\{\left(\mathrm{H}_{2} \mathrm{O}\right) \mathrm{Fe}(\mathrm{L})\right\}\right.$ $\left.\left\{\mathrm{Nb}(\mathrm{CN})_{8}\right\}\{\mathrm{Fe}(\mathrm{L})\}\right]_{\infty}$ has been synthesized [16]. Its crystal structure studies reveal that it consists of topologically quasi-one dimensional spin chains with helical geometry. This system shows SCM character of slow relaxation and large DC susceptibility at low temperatures. The back bone of each chain consists of alternate $\mathrm{Fe}$ and $\mathrm{Nb}$ ions with each $\mathrm{Nb}$ ion connected to an additional off-chain $\mathrm{Fe}$ ion (see Fig 1). $\mathrm{Nb}$ ions have spin 1/2 while $\mathrm{Fe}$ ions have spin of 2. The coordination around $\mathrm{Fe}(I I)$ is the unusual hepta coordination which leads to large anisotropy of the $\mathrm{Fe}$ spins. All the intrachain interactions are antiferromagnetic and much stronger than interchain interaction. Since the $F e$ ions have large and negative (easy-axis) anisotropy, we can assume the $F e$ spins to be Ising-type, with non-collinear spin orientations due to helicity of the chain. As the spin axes are non-collinear, different components of $N b$ spins are involved in interactions at different sites along the helix and therefore must be treated quantum mechanically. A careful examination of the chain structure, shows that two chemical units form a geometrical unit which are used as basic blocks in our transfer matrix method. To define this complex structural unit, we introduce three structural parameters, $\omega, \alpha$ and $\delta$, which are defined in Fig (1). We note that, here a quantum unit is formed by two $\mathrm{Nb}$ ions connected by one in-chain $\mathrm{Fe}$ ion and each of the $\mathrm{Nb}$ ions being connected to an off-chain $\mathrm{Fe}$ ion. These quantum units are connected by in-chain $\mathrm{Fe}$ ions, which behave like classical spins. Therefore, this system falls 


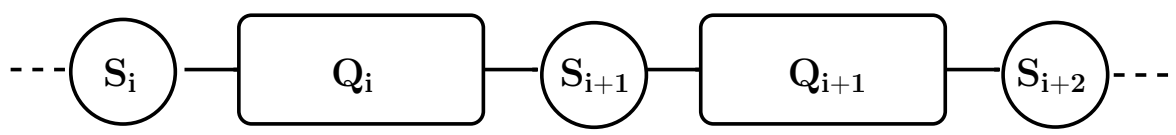

Figure 2: Schematic diagram of a chain with alternative quantum and classical units

into the class of SCMs we discussed in the previous section. In Sec 4, we first discuss in detail the application of our technique to solve the spin model for the SCM system. This is followed by presentation of results and their comparison with experimental studies.

\section{Description of the Technique}

Let us consider our system formed by identical quantum units connected by classical (Isingtype) spins (see Fig 2). Let $\mathbf{H}_{i}^{Q M}\left(\mathbf{Q}_{i}\right)$ be the Hamiltonian associated with $i$-th quantum unit with the set of quantum and classical spin operators $\mathbf{Q}_{i}$. Let the Hamiltonian for the interaction of the $i$-th quantum unit with classical spin, $\mathbf{S}_{i}$, to the left, be $\mathbf{H}_{i}^{I, L}\left(\mathbf{q}_{i}^{L}, \mathbf{S}_{i}\right)$ with $\mathbf{q}_{i}^{L}$ the quantum spin operator of the $i$-th quantum unit involved in interaction to the left. We can similarly define $\mathbf{H}_{i}^{I, R}\left(\mathbf{q}_{i}^{R}, \mathbf{S}_{i+1}\right)$ as the Hamiltonian for the interaction of the $i$-th quantum unit with classical spin, $S_{i+1}$, to the right. In the presence of (uniform) external magnetic field $\vec{B}$, there will be Zeeman interaction term $-g \mu_{B} \mathbf{S}_{Z, i} B$, where $\mathbf{S}_{Z, i}$ be the $Z$-component of spin for the $i$-th block, and this term is here interpreted as sum of the projection of spin for the $i$-th quantum unit and half of that for both $i$-th and $(i+1)$-th classical spins along the global (laboratory) $Z$-axis. Here $\mathbf{S}_{Z, i}$ is defined with weight $1 / 2$ for the classical spins to avoid double counting. We now can write our total Hamiltonian of a chain as, $\mathbf{H}=\sum_{i=1}^{N} \mathbf{H}_{i}$, where,

$$
\mathbf{H}_{i}=\mathbf{H}_{i}^{I, L}\left(\mathbf{q}_{i}^{L}, \mathbf{S}_{i}\right)+\mathbf{H}_{i}^{Q M}\left(\mathbf{Q}_{i}\right)+\mathbf{H}_{i}^{I, R}\left(\mathbf{q}_{i}^{R}, \mathbf{S}_{i+1}\right)-g \mu_{B} \mathbf{S}_{Z, i} B,
$$

In the last term, the $g$-factor is assumed to be an average over all the corresponding $g$-factors of different spin-sites and $\mu_{B}$ is the Bohr magneton. We note that, the Zeeman interaction term has three different parts: $\frac{1}{2} \mathbf{H}_{i}^{Z m}\left(\mathbf{S}_{i}, \vec{B}\right), \mathbf{H}_{i}^{Z m}\left(\mathbf{Q}_{i}, \vec{B}\right)$ and $\frac{1}{2} \mathbf{H}_{i+1}^{Z m}\left(\mathbf{S}_{i+1}, \vec{B}\right)$ corresponding to the one associated with $i$-th classical spin, $i$-th quantum unit and $(i+1)$-th classical spin respectively. To avoid double counting, as before, Zeeman interaction part for $i$-th and $(i+1)$-th classical spins have been taken with weights $1 / 2$. Partition function for the chain (having $N$ quantum and classical units) at temperature $T$ can be written as,

$$
Q_{N}(\beta, B)=\operatorname{Tr} e^{-\beta \mathbf{H}}
$$

where, 'Tr' means trace and $\beta=1 /\left(k_{B} T\right), k_{B}$ being the Boltzmann constant. Now let us consider direct product basis of the whole chain. This can simply be written as, $\left|\cdots, \sigma_{i}, Q_{i}, \cdots\right\rangle$, where $\left|\sigma_{i}\right\rangle$ and $\left|Q_{i}\right\rangle$ represents convenient basis for $i$-th classical spin and quantum unit, respectively. Eq (2) can now be written as,

$$
Q_{N}(\beta, B)=\sum_{\{\sigma, Q\}}\left\langle\cdots, \sigma_{i}, Q_{i}, \cdots\left|e^{-\beta \mathbf{H}}\right| \cdots, \sigma_{i}, Q_{i}, \cdots\right\rangle
$$


Here the sums run over all possible configurations $\{\sigma, Q\}$ of all the classical spins and the quantum units. Now since, $\mathbf{H}_{i}$ and $\mathbf{H}_{j}$ commute, we can rewrite Eq (3) in the following form:

$$
\begin{aligned}
Q_{N}(\beta, B) & =\sum_{\{\sigma, Q\}}\left\langle\cdots, \sigma_{i}, Q_{i}, \cdots\left|\prod_{i=1}^{N} e^{-\beta \mathbf{H}_{i}}\right| \cdots, \sigma_{i}, Q_{i}, \cdots\right\rangle \\
& =\sum_{\{\sigma\}}\left\langle\cdots, \sigma_{i}, \cdots\left|\prod_{i=1}^{N}\left(\sum_{Q_{i}}\left\langle Q_{i}\left|e^{-\beta \mathbf{H}_{i}}\right| Q_{i}\right\rangle\right)\right| \cdots, \sigma_{i}, \cdots\right\rangle
\end{aligned}
$$

Here, $Q_{i}$ is the set of configurations for $i$-th quantum unit. Note, the quantity in parentheses, $\sum_{Q_{i}}\left\langle Q_{i}\left|e^{-\beta \mathbf{H}_{i}}\right| Q_{i}\right\rangle$, is a purely classical operator (containing $\mathbf{S}_{i}$ and $\mathbf{S}_{i+1}$ only) as we have summed over quantum variables of the unit. Denoting it as $\mathbf{T}_{i}$ enables us to rewrite Eq (4) as,

$$
Q_{N}(\beta, B)=\sum_{\{\sigma\}}\left\langle\cdots, \sigma_{i}, \cdots\left|\prod_{i=1}^{N} \mathbf{T}_{i}\right| \cdots, \sigma_{i}, \cdots\right\rangle
$$

This form is familiar to us, with $\mathbf{T}_{i}$ being the transfer operator. Introducing the identity $\sum_{\sigma_{i}}\left|\sigma_{i}\right\rangle\left\langle\sigma_{i}\right|$ between successive Ts, will enable us to write the Partition function as a trace of $N$-th power of a transfer matrix, $\mathbf{P}$,

$$
Q_{N}(\beta, B)=\operatorname{tr} \mathbf{P}^{N}
$$

where, elements of the transfer matrix $\mathbf{P}$ are given by,

$$
P_{\sigma_{i}, \sigma_{i+1}}=\left\langle\sigma_{i}\left|\mathbf{T}_{i}\right| \sigma_{i+1}\right\rangle
$$

Since we have taken classical spins to be Ising-type (which can have only two values, \pm 1 ), the $\mathbf{P}$ matrix will be a $2 \times 2$ matrix. Diagonalizing this matrix is trivial; let $\lambda_{+}$and $\lambda_{-}$be the two eigenvalues of the matrix, then they can be expressed in terms of four elements of $\mathbf{P}$ as: $\lambda_{ \pm}=\frac{1}{2}\left[\left(p_{11}+p_{22}\right) \pm \sqrt{\left(p_{11}-p_{22}\right)^{2}+4 p_{12} p_{21}}\right]$. In terms of eigenvalues, the partition function can now be written as,

$$
Q_{N}(\beta, B)=\lambda_{+}^{N}+\lambda_{-}^{N}
$$

Note both the eigenvalues are function of $\beta$ and $B$. For thermodynamically large $N$, one can only take the larger eigenvalue (here $\lambda_{+}$), but for finite $N$, one should take both of them to evaluate the partition function. $\chi T$ as a function of $T$ ( $\chi$ being susceptibility), can be obtained as,

$$
\chi T=\frac{N_{A} k_{B} T^{2}}{N} \frac{\partial^{2}}{\partial B^{2}} \ln Q_{N}(\beta, B)
$$

where, $N_{A}$ is the Avogadro's number. In Eq (9), 2nd order differentiation can be done easily, one can even get simple closed analytical form for small applied magnetic field $B$. In general, one can do simple numerical differentiation to get $\chi T$ value as a function of temperature $T$. 
We now need to discuss a little more about $\mathbf{T}_{i}$. If we take basis $\left|Q_{i}\right\rangle$ to be eigenstates of $\mathbf{H}_{i}$, then $\mathbf{T}_{i}$ will just be sum of exponential of those eigenvalues. Let by diagonalizing it (if quantum unit has unconnected spin-1/2 quantum spins, we can do it trivially and get analytical expression) we get eigenvalues $L_{j}^{i}$, where $j$ runs from 1 to $d$ (number of basis vectors or dimensionality of configurational space for the quantum unit). Note these eigenvalues are functions of only $\mathbf{S}_{i}$ and $\mathbf{S}_{i+1}$, the classical spin operators. So we can write $\mathbf{T}_{i}$ in the following way:

$$
\begin{aligned}
\mathbf{T}_{i} & =\sum_{Q_{i}}\left\langle Q_{i}\left|e^{-\beta \mathbf{H}_{i}}\right| Q_{i}\right\rangle \\
& =\sum_{j=1}^{d} e^{-\beta L_{j}^{i}}
\end{aligned}
$$

In our case $\left(\mathrm{Fe}_{2} \mathrm{Nb}\right)$, quantum unit has five spins (3 Ising spins and 2 quantum spin-1/2s), hence $d=32$ and $\mathbf{T}_{i}$ will involve summation over the 32 eigenvalues.

\subsection{Consideration of Interchain Interaction}

Interchain interaction is an important issue in this field of SCMs; in real systems it is not possible to separate chains to eliminate this interaction. With increase in this interaction strength, a one dimensional system like SCM gradually transforms into a higher dimensional system with remarkably different properties. Even a weak interchain interaction can change properties of a chain -its presence can be seen both in static (like $\chi T$ vs.T plots, hysteresis curves) and dynamic (like, Cole-Cole plots) measurements. Unfortunately, there is no detailed and explicit discussion on how one can treat this interaction theoretically. In fact, this issue has been highlighted in a paper by Miyasaka et al. [2], where it is posed as an open problem. In this section we discuss interchain interaction in detail.

In low-dimension, the quantum fluctuations are generally large. However SCMs are not strictly one-dimensional, and we have weak interchain interaction. It is customary to deal with interchain interactions within a mean field approximation. Here a widely used formula for corrected (or modified) susceptibility due to the interaction is $\chi_{M}=\chi_{0} /\left(1+z J^{\prime} \chi_{0} / N_{A} g^{2} \mu_{B}^{2}\right)$, where $\chi_{0}$ is susceptibility of the chain without interchain interactions, $z$ is the number of nearest neighbors and $J^{\prime}$ is interchain interaction strength ( $z J^{\prime}$ together is called mean field parameter) [17, 18, 19]. However we wish to emphasize that, this formula is for vanishing applied magnetic field. So in case of finite magnetic field and at very low temperatures this formula is not appropriate. In addition, for a chain, unlike for molecular systems, $S_{Z}$ can be very large; so it is not clear whether it is valid to make series expansion of exponential function of the Zeeman and interchain interactions and retain terms only up to first order. Here we discuss a mean-field approach where the interaction is dealt by transfer matrix technique and can be used to study the case when magnetic field is finite. We also wish to make it clear that, there has to be a factor $N$ (number of chemical units in a chain) multiplying $z J^{\prime}$ in the above formula of modified susceptibility for vanishing magnetic field. To the best of our knowledge, this is the first attempt to deal with interchain interactions by the transfer matrix technique. 
If the interchain interaction is weak, we can bring in the effect of environment by placing a chain in the mean field generated by neighboring chains. Let us now consider $\left\langle S_{Z}\right\rangle$ be the expectation value of $Z$-component of spin for each chain and $z J^{\prime}$ be the interchain interaction (mean field) parameter, then the interaction energy can be written as: $z J^{\prime} \mathbf{S}_{Z}\left\langle S_{Z}\right\rangle$, with $\mathbf{S}_{Z}$ being the $Z$-component of total spin for a chain. Now since $\mathbf{S}_{Z}=\sum_{i=1}^{N} \mathbf{S}_{Z, i}$ with $\mathbf{S}_{Z, i}$ being the $Z$-component of spin for $i$-th block, we can rewrite the interaction energy as $\sum_{i=1}^{N}\left(z J^{\prime} \mathbf{S}_{Z, i}\left\langle S_{Z}\right\rangle\right)$, which can be viewed as sum of interaction energy of each block of a chain with a mean field generated by neighboring chains. Now if we add this mean field block interaction energy term, $z J^{\prime} \mathbf{S}_{Z, i}\left\langle S_{Z}\right\rangle$, to $\mathbf{H}_{i}$ of Eq (1), we get,

$$
\begin{aligned}
\mathbf{H}_{i} & =\mathbf{H}_{i}^{I, L}\left(\mathbf{q}_{i}^{L}, \mathbf{S}_{i}\right)+\mathbf{H}_{i}^{Q M}\left(\mathbf{Q}_{i}\right)+\mathbf{H}_{i}^{I, R}\left(\mathbf{q}_{i}^{R}, \mathbf{S}_{i+1}\right)-g \mu_{B} \mathbf{S}_{Z, i} B+z J^{\prime} \mathbf{S}_{Z, i}\left\langle S_{Z}\right\rangle \\
& =\mathbf{H}_{i}^{I, L}\left(\mathbf{q}_{i}^{L}, \mathbf{S}_{i}\right)+\mathbf{H}_{i}^{Q M}\left(\mathbf{Q}_{i}\right)+\mathbf{H}_{i}^{I, R}\left(\mathbf{q}_{i}^{R}, \mathbf{S}_{i+1}\right)-g \mu_{B} \mathbf{S}_{Z, i} B^{\prime}
\end{aligned}
$$

where, $B^{\prime}=B-\frac{z J^{\prime}}{g \mu_{B}}\left\langle S_{Z}\right\rangle$. We can then follow the usual transfer matrix technique and get a partition function for the chain in the presence of a modified magnetic field $B^{\prime}$. Since, $\left\langle S_{Z}\right\rangle=$ $\frac{1}{g \mu_{B} \beta} \frac{\partial \ln Q_{N}\left(\beta, B^{\prime}\right)}{\partial B^{\prime}}$, we see that, $\left\langle S_{Z}\right\rangle$ has to be solved self-consistently by an iterative procedure. We can use the following self-consistent scheme developed by first noting that, $z J^{\prime}$ being very small, internal field, $\Delta B=-\frac{z J^{\prime}}{g \mu_{B}}\left\langle S_{Z}\right\rangle$, is a slowly varying function of external applied field $B$ resulting in $\left|\frac{\partial \Delta B}{\partial B}\right| \ll 1$ or $\left|\frac{\partial B^{\prime}}{\partial B}\right| \approx 1$. Using the conditions and making the Taylor series expansion of $\ln Q_{N}\left(\beta, B^{\prime}\right)$ about $B$, we get,

$$
\begin{aligned}
g \mu_{B} \beta\left\langle S_{Z}\right\rangle= & \frac{\partial \ln Q_{N}\left(\beta, B^{\prime}\right)}{\partial B^{\prime}} \\
\simeq & \frac{\partial \ln Q_{N}(\beta, B)}{\partial B}-\left(\frac{z J^{\prime}}{g \mu_{B}}\right) \frac{\partial^{2} \ln Q_{N}(\beta, B)}{\partial B^{2}}\left\langle S_{Z}\right\rangle+ \\
& \frac{1}{2}\left(\frac{z J^{\prime}}{g \mu_{B}}\right)^{2} \frac{\partial^{3} \ln Q_{N}(\beta, B)}{\partial B^{3}}\left\langle S_{Z}\right\rangle^{2}-\cdots
\end{aligned}
$$

Iteration can be started with $\left\langle S_{Z}\right\rangle=\frac{1}{g \mu_{B} \beta} \frac{\partial \ln Q_{N}(\beta, B)}{\partial B}$, without interchain interaction, finally leading to a converged $\left\langle S_{Z}\right\rangle$ value. The self-consistent result correct to second order in $z J^{\prime}$ is given by,

$$
\begin{aligned}
g \mu_{B}\left\langle S_{Z}\right\rangle= & M(\beta, B)-\left(\frac{z J^{\prime}}{g^{2} \mu_{B}^{2}}\right) M(\beta, B) \frac{\partial M(\beta, B)}{\partial B}+ \\
& \left(\frac{z J^{\prime}}{g^{2} \mu_{B}^{2}}\right)^{2}\left[M(\beta, B)\left(\frac{\partial M(\beta, B)}{\partial B}\right)^{2}+\frac{1}{2}(M(\beta, B))^{2} \frac{\partial^{2} M(\beta, B)}{\partial B^{2}}\right]
\end{aligned}
$$

where, $M(\beta, B)=\frac{1}{\beta} \frac{\partial \ln Q_{N}(\beta, B)}{\partial B}$ is the magnetization in the absence of interchain interaction. Going to higher order in $z J^{\prime}$ is possible but generally not necessary. Obtaining modified susceptibility $\left(\chi_{M}\right)$ from Eq (13) is straight forward. In the vanishing field regime, we can get 
the widely used formula for $\chi_{M}$ by retaining terms up to first order in $z J^{\prime}$ of the Eq (12) and subsequently solving for $\left\langle S_{Z}\right\rangle$. This gives,

$$
g \mu_{B}\left\langle S_{Z}\right\rangle=\frac{\frac{1}{\beta} \frac{\partial \ln Q_{N}(\beta, B)}{\partial B}}{1+\frac{z J^{\prime}}{g^{2} \mu_{B}^{2} \beta} \frac{\partial^{2} \ln Q_{N}(\beta, B)}{\partial B^{2}}}
$$

Now in this regime, modified susceptibility is $\chi_{M}=\frac{N_{A}}{B N} g \mu_{B}\left\langle S_{Z}\right\rangle$ and susceptibility without interchain interaction is $\chi_{0}=\frac{N_{A}}{B N \beta} \frac{\partial \ln Q_{N}(\beta, B)}{\partial B}$. This directly leads to:

$$
\chi_{M}=\frac{\chi_{0}}{1+\frac{z J^{\prime} N}{g^{2} \mu_{B}^{2} N_{A}} \chi_{0}}
$$

Note the difference of factor of $N$ with this formula and the one generally used in this field [17, 18, 19]. Appearance of this factor in Eq (15) is easy to understand: while susceptibility is measured for Avogadro number $\left(N_{A}\right)$ of chemical units, in the transfer matrix method we compute partition function for $N$ (chemical) units; therefore, we need to normalize the susceptibility computed from the partition function. The factor $N z J^{\prime}$ can be given a physical interpretation. Let us assume that a unit in a given chain interacts only with the corresponding units of the $z$ neighboring chains and $p$ be the total effective coupling strength, then the associated interaction term will be $p \mathbf{S}_{Z, i} \frac{\left\langle S_{Z}\right\rangle}{N}$, where $\frac{\left\langle S_{Z}\right\rangle}{N}$ is the expectation value of $Z$-component of spin for a unit. The total mean field interaction term is therefore given by $\sum_{i} p \mathbf{S}_{Z, i} \frac{\left\langle S_{Z}\right\rangle}{N}$. Comparing this with conventional chain-chain total interaction term $\sum_{i} z J^{\prime} \mathbf{S}_{Z, i}\left\langle S_{Z}\right\rangle$, we get $p=N z J^{\prime}$. Thus, while $z J^{\prime}$ is the coupling strength of a chain with its $z$ neighboring chains, $N z J^{\prime}$ is the coupling strength of a given unit with the corresponding units of the $z$ neighboring chains. We will report the latter parameter $\left(N z J^{\prime}\right)$ for our study of real system.

This way of introducing interchain interaction in computing magnetic susceptibility is fairly general and can be applied to any class of SCMs. Here we have presented a formula for modified susceptibility which is very similar to the one used for molecular systems and generally adopted for SCMs. The higher order self-consistent method (Eq 13) is more general and applicable even when the magnetic field is not vanishingly small.

\section{Application to the $\mathrm{Fe}_{2} \mathrm{Nb}$ system}

In this section we will apply our technique to the system $\mathrm{Fe}_{2} \mathrm{Nb}$ which is already introduced in Sec. 2. All the $\mathrm{Fe}-\mathrm{Nb}$ couplings are supposed to be antiferromagnetic and are assumed to be of same strength $(J)$. We assume that the strength of axial anisotropy constant $D$ to be the same for each $F e$ ions. As we already mentioned, since this $D$ is known to be large and negative, we treat the spins on $F e$ ions as two state Ising spins along their respective anisotropy axis. We also further assume the $g$-factor to be isotropic and has same value for all the ions. Three parameters are defined in Fig (1), namely, $\omega, \alpha$ and $\delta$, to describe complex structural unit of the chain. In Eq (9) we need to use average partition function, averaged over all possible 
orientations of a chain to compare with experiments done on a powder sample. The averaging can be done by noting that a chain orientation in space can be defined by three angles, namely, $\theta, \phi$ and $\psi$. Here $\theta$ and $\phi$ are usual spherical polar coordinates to define the orientation of the chain axis and $\psi$ is measure of rotation of the chain about its own axis. Our partition function will depend on (a) temperature, (b) magnetic field and (c) all three structural parameters $(\omega, \alpha$ and $\delta$ )for a single chain, it will also depend on orientational angles $(\theta, \phi$ and $\psi)$ with respect to laboratory frame. The partition function can be averaged over all orientations of the chain axis to obtain an average partition function $\bar{Q}_{N}(\beta, B)$, given by,

$$
\bar{Q}_{N}(\beta, B)=\int_{\theta=0}^{\pi} \int_{\phi=0}^{2 \pi} \int_{\psi=0}^{2 \pi} Q_{N}(\beta, B ; \theta, \phi, \psi) \sin \theta d \theta d \phi d \psi
$$

where, $Q_{N}(\beta, B ; \theta, \phi, \psi)$ is the partition function of the chain for a particular orientation $(\theta, \phi, \psi)$. We have ignored a normalization constant, as it will not affect our result. Integrations in Eq (16) can be done by simple numerical methods (analytically it may not always be possible). So in this particular problem, Eq (9) must be replaced by,

$$
\chi T=\frac{N_{A} k_{B} T^{2}}{2 N} \frac{\partial^{2}}{\partial B^{2}} \ln \bar{Q}_{N}(\beta, B)
$$

Note, we have also replaced $N$ by $2 N$, as one structural unit consists of two chemical units and susceptibility from experiment is quoted for an Avogadro number of chemical units.

The Hamiltonian for $i$-th quantum unit consisting of $N b_{1}, F e_{1}^{\prime}, F e_{c}, N b_{2}$ and $F e_{2}^{\prime}$ site spins and connected to neighboring quantum units by $F e_{1}$ and $F e_{2}$ site spins respectively, can be written as:

$$
\begin{aligned}
\mathbf{H}_{i}= & J \overrightarrow{\mathbf{S}}^{F e_{1}} \cdot \overrightarrow{\mathbf{S}}^{N b_{1}}+J \overrightarrow{\mathbf{S}}^{F e_{2}} \cdot \overrightarrow{\mathbf{S}}^{N b_{2}}+J\left(\overrightarrow{\mathbf{S}}^{F e_{1}^{\prime}} \cdot \overrightarrow{\mathbf{S}}^{N b_{1}}+\overrightarrow{\mathbf{S}}^{F e_{c}} \cdot \overrightarrow{\mathbf{S}}^{N b_{1}}+\overrightarrow{\mathbf{S}}^{F e_{c}} \cdot \overrightarrow{\mathbf{S}}^{N b_{2}}+\overrightarrow{\mathbf{S}}^{F e_{2}^{\prime}} \cdot \overrightarrow{\mathbf{S}}^{N b_{2}}\right) \\
& -\frac{1}{2} g \mu_{B} \overrightarrow{\mathbf{S}}^{F e_{1}} \cdot \vec{B}-\frac{1}{2} g \mu_{B} \overrightarrow{\mathbf{S}}^{F e_{2}} \cdot \vec{B} \\
& -g \mu_{B}\left(\overrightarrow{\mathbf{S}}^{N b_{1}} \cdot \vec{B}+\overrightarrow{\mathbf{S}}^{F e_{1}^{\prime}} \cdot \vec{B}+\overrightarrow{\mathbf{S}}^{F e_{c}} \cdot \vec{B}+\overrightarrow{\mathbf{S}}^{N b_{2}} \cdot \vec{B}+\overrightarrow{\mathbf{S}}^{F e_{2}^{\prime}} \cdot \vec{B}\right)
\end{aligned}
$$

Here $\overrightarrow{\mathbf{S}}$ is a vector spin operator for the specified ion. All these spin operators are defined relative to their corresponding local coordinate systems (see Fig 1). For example, $z$-axis of a $\mathrm{Fe}$ ion is taken along its easy anisotropy axis. First and second terms in Eq (18) are interaction terms between quantum unit and its neighboring classical units. The 3 rd term within brackets includes all the interactions within the quantum unit. The 4th, 5th and 6th terms are the Zeeman terms associated with classical spins with appropriate weight and the quantum unit respectively. All these terms can be expressed explicitly with the help of structural angles or parameters $(\omega, \alpha, \delta)$ and polar coordinates of the chain $(\theta, \phi, \psi)$. Table (1) gives the expression for each of the terms in the laboratory frame assuming that the magnetic field is applied along $Z$-axis of the laboratory frame. Knowing all these interaction terms, now we can form the transfer operator $\mathbf{T}$ for the $i$-th unit as,

$$
\begin{aligned}
\mathbf{T}_{i} & =\sum_{\left\{Q_{i}\right\}}\left\langle Q_{i}\left|e^{-\beta \mathbf{H}_{i}}\right| Q_{i}\right\rangle \\
& =\sum_{\left\{Q_{i}\right\}}\left\langle Q_{i}\left|e^{\left(a \mathbf{S}_{x}^{N b_{1}}+b \mathbf{S}_{y}^{N b_{1}}+c \mathbf{S}_{z}^{N b_{1}}\right)} e^{\left(d \mathbf{S}_{x}^{N b_{2}}+e \mathbf{S}_{y}^{N b_{2}}+f \mathbf{S}_{z}^{N b_{2}}\right)} e^{h}\right| Q_{i}\right\rangle
\end{aligned}
$$


Table 1: Expression for all 13 interaction terms appearing in Eq (18) is given below. Treating the $F e$ spins as Ising spins with anisotropy along $z$ direction, we replace the spin by $2 \sigma^{F e_{1}}$, where $\sigma^{F e_{1}}$ is the Ising-variable with only possible values \pm 1 , the factor 2 is to account for the spin of the $\mathrm{Fe}$ ion which is 2 .

\begin{tabular}{|c|c|}
\hline Term & Expression \\
\hline $\overrightarrow{\mathbf{S}}^{F e_{1}} \cdot \overrightarrow{\mathbf{S}}^{N b_{1}}$ & $2 \sigma^{F e_{1}}\left(\mathbf{S}_{x}^{N b_{1}} \cos \omega \cos \alpha+\mathbf{S}_{y}^{N b_{1}} \sin \omega+\mathbf{S}_{z}^{N b_{1}} \cos \omega \sin \alpha\right)$ \\
\hline $\overrightarrow{\mathbf{S}}^{F e_{2}} \cdot \overrightarrow{\mathbf{S}}^{N b_{2}}$ & $2 \sigma^{F e_{2}}\left(\mathbf{S}_{x}^{N b_{2}} \cos \omega \cos \alpha-\mathbf{S}_{y}^{N b_{2}} \sin \omega+\mathbf{S}_{z}^{N b_{2}} \cos \omega \sin \alpha\right)$ \\
\hline $\overrightarrow{\mathbf{S}}^{F e_{1}^{\prime}} \cdot \overrightarrow{\mathbf{S}}^{N b_{1}}$ & $2 \sigma^{F e_{1}^{\prime}}\left(\mathbf{S}_{z}^{N b_{1}} \cos \delta+\mathbf{S}_{y}^{N b_{1}} \sin \delta\right)$ \\
\hline $\overrightarrow{\mathbf{S}}^{F e_{c}} \cdot \overrightarrow{\mathbf{S}}^{N b_{1}}$ & $2 \sigma^{F e_{c}}\left(\mathbf{S}_{x}^{N b_{1}} \cos \omega \cos \alpha-\mathbf{S}_{y}^{N b_{1}} \sin \omega+\mathbf{S}_{z}^{N b_{1}} \cos \omega \sin \alpha\right)$ \\
\hline $\overrightarrow{\mathbf{S}}^{F e_{c}} \cdot \overrightarrow{\mathbf{S}}^{N b_{2}}$ & $2 \sigma^{F e_{c}}\left(\mathbf{S}_{x}^{N b_{2}} \cos \omega \cos \alpha+\mathbf{S}_{y}^{N b_{2}} \sin \omega+\mathbf{S}_{z}^{N b_{2}} \cos \omega \sin \alpha\right)$ \\
\hline $\overrightarrow{\mathbf{S}}^{F e_{2}^{\prime}} \cdot \overrightarrow{\mathbf{S}}^{N b_{2}}$ & $2 \sigma^{F e_{2}^{\prime}}\left(\mathbf{S}_{z}^{N b_{2}} \cos \delta+\mathbf{S}_{y}^{N b_{2}} \sin \delta\right)$ \\
\hline $\overrightarrow{\mathbf{S}} F e_{1} \cdot \vec{B}$ & $2 \sigma^{F e_{1}}(\cos \omega \cos \theta+\sin \omega \sin \psi \sin \theta) B$ \\
\hline $\overrightarrow{\mathbf{S}^{F e_{2}} \cdot \vec{B}}$ & $2 \sigma^{F e_{2}}(\cos \omega \cos \theta+\sin \omega \sin \psi \sin \theta) B$ \\
\hline $\overrightarrow{\mathbf{S}}^{N b_{1}} \cdot \vec{B}$ & $\begin{array}{l}{\left[\mathbf{S}_{x}^{N b_{1}}(\cos \alpha \cos \theta-\sin \alpha \sin \theta \cos \psi)+\mathbf{S}_{y}^{N b_{1}} \sin \psi \sin \theta\right.} \\
\left.+\mathbf{S}_{z}^{N b_{1}}(\sin \alpha \cos \theta+\cos \alpha \sin \theta \cos \psi)\right] B\end{array}$ \\
\hline $\overrightarrow{\mathbf{S}}^{F e_{1}^{\prime}} \cdot \vec{B}$ & $2 \sigma^{F e_{1}^{\prime}}(\sin \delta \sin \psi \sin \theta+\cos \delta \sin \alpha \cos \theta+\cos \delta \cos \alpha \cos \psi \sin \theta) B$ \\
\hline $\overrightarrow{\mathbf{S}}^{F e_{c}} \cdot \vec{B}$ & $2 \sigma^{F e_{c}}(\cos \omega \cos \theta-\sin \omega \sin \psi \sin \theta) B$ \\
\hline $\overrightarrow{\mathbf{S}}^{N b_{2}} \cdot \vec{B}$ & $\begin{array}{l}{\left[\mathbf{S}_{x}^{N b_{2}}(\cos \alpha \cos \theta+\sin \alpha \sin \theta \cos \psi)-\mathbf{S}_{y}^{N b_{2}} \sin \psi \sin \theta\right.} \\
\left.+\mathbf{S}_{z}^{N b_{2}}(\sin \alpha \cos \theta-\cos \alpha \sin \theta \cos \psi)\right] B\end{array}$ \\
\hline $\overrightarrow{\mathbf{S}}^{F e_{2}^{\prime}} \cdot \vec{B}$ & $2 \sigma^{F e_{2}^{\prime}}(-\sin \delta \sin \psi \sin \theta+\cos \delta \sin \alpha \cos \theta-\cos \delta \cos \alpha \cos \psi \sin \theta) B$ \\
\hline
\end{tabular}


Expressions for $a, b, c, d, e, f$ and $h$, which are functions of classical spins involved in the problem, are given in Table (2). Here $\left|Q_{i}\right\rangle$ is the direct product basis of the spins of $N b_{1}$,

Table 2: Expression for variables appeared in Eq (19)

\begin{tabular}{|c|l|}
\hline Variable & Expression \\
\hline$a$ & $-\beta\left[2 J\left(\sigma^{F e_{1}}+\sigma^{F e_{c}}\right) \cos \omega \cos \alpha-g \mu_{B} B(\cos \alpha \cos \theta-\sin \alpha \sin \theta \cos \psi)\right]$ \\
\hline$b$ & $-\beta\left[2 J\left\{\left(\sigma^{F e_{1}}-\sigma^{F e_{c}}\right) \sin \omega+\sigma^{F e_{1}^{\prime}} \sin \delta\right\}-g \mu_{B} B \sin \psi \sin \theta\right]$ \\
\hline$c$ & $-\beta\left[2 J\left\{\left(\sigma^{F e_{1}}+\sigma^{F e_{c}}\right) \cos \omega \sin \alpha+\sigma^{F e_{1}^{\prime}} \cos \delta\right\}-g \mu_{B} B(\sin \alpha \cos \theta+\cos \alpha \sin \theta \cos \psi)\right]$ \\
\hline$d$ & $-\beta\left[2 J\left(\sigma^{F e_{c}}+\sigma^{F e_{2}}\right) \cos \omega \cos \alpha-g \mu_{B} B(\cos \alpha \cos \theta+\sin \alpha \sin \theta \cos \psi)\right]$ \\
\hline$e$ & $-\beta\left[2 J\left\{\left(\sigma^{F e_{c}}-\sigma^{F e_{2}}\right) \sin \omega+\sigma^{F e_{2}^{\prime}} \sin \delta\right\}+g \mu_{B} B \sin \psi \sin \theta\right]$ \\
\hline$f$ & $-\beta\left[2 J\left\{\left(\sigma^{F e_{c}}+\sigma^{F e_{2}}\right) \cos \omega \sin \alpha+\sigma^{F e_{2}^{\prime}} \cos \delta\right\}-g \mu_{B} B(\sin \alpha \cos \theta-\cos \alpha \sin \theta \cos \psi)\right]$ \\
\hline$h$ & $\beta g \mu_{B} B\left[\left(\sigma^{F e_{1}}+\sigma^{F e_{2}}\right)(\cos \omega \cos \theta+\sin \omega \sin \theta \sin \psi)\right.$ \\
& $+2 \sigma^{F e_{1}^{\prime}}(\sin \delta \sin \theta \sin \psi+\cos \delta \sin \alpha \cos \theta+\cos \delta \cos \alpha \sin \theta \cos \psi)$ \\
& $+2 \sigma^{F e_{c}}(\cos \omega \cos \theta-\sin \omega \sin \theta \sin \psi)$ \\
& $\left.+2 \sigma^{F e_{2}^{\prime}}(-\sin \delta \sin \theta \sin \psi+\cos \delta \sin \alpha \cos \theta-\cos \delta \cos \alpha \sin \theta \cos \psi)\right]$ \\
\hline
\end{tabular}

$F e_{1}^{\prime}, F e_{c}, N b_{2}$, and $F e_{2}^{\prime}$. If we take the basis for $N b_{1}$ to be eigenstates of $\left(a \mathbf{S}_{x}^{N b_{1}}+b \mathbf{S}_{y}^{N b_{1}}+\right.$ $c \mathbf{S}_{z}^{N b_{1}}$ ) (the quantum mechanical operator in the exponent of the first exponential in Eq (19)), the corresponding exponential term will be reduced to sum of exponential of the eigenvalues of the operator. Similarly second exponential term can also be reduced to the sum of exponential of the eigenvalues of the corresponding quantum operator $\left(d \mathbf{S}_{x}^{N b_{2}}+e \mathbf{S}_{y}^{N b_{2}}+f \mathbf{S}_{z}^{N b_{2}}\right)$. Finding eigenvalues of these quantum mechanical operators is not difficult; they are $\pm \lambda^{N b_{1}}$ and $\pm \lambda^{N b_{2}}$ respectively, where, $\lambda^{N b_{1}}=\frac{1}{2} \sqrt{a^{2}+b^{2}+c^{2}}$ and $\lambda^{N b_{2}}=\frac{1}{2} \sqrt{d^{2}+e^{2}+f^{2}}$. We now write the transfer operator in Eq (19) as,

$$
\begin{aligned}
\mathbf{T}_{i} & =\sum_{\left\{Q_{i}^{\prime}\right\}}\left(e^{\lambda^{N b_{1}}}+e^{-\lambda^{N b_{1}}}\right)\left(e^{\lambda^{N b_{2}}}+e^{-\lambda^{N b_{2}}}\right) e^{h} \\
& =4 \sum_{\left\{Q_{i}^{\prime}\right\}} \cosh \lambda^{N b_{1}} \cosh \lambda^{N b_{2}} e^{h}
\end{aligned}
$$

where the sum runs over all the configurations of the classical spins, $\left\{\sigma^{F e_{1}^{\prime}}, \sigma^{F e_{c}}, \sigma^{F e_{2}^{\prime}}\right\}$, within the quantum unit $i$, which is denoted by $\left\{Q_{i}^{\prime}\right\}$. Note, $\mathbf{T}_{i}$ depends only on $\sigma^{\mathrm{Fe}_{1}}$ and $\sigma^{\mathrm{Fe}_{2}}$ and we can write elements of transfer matrix $\mathbf{P}$ as:

$$
p_{\sigma^{F e_{1}}, \sigma^{F e_{2}}}=4 \sum_{\left\{Q_{i}^{\prime}\right\}} \cosh \lambda^{N b_{1}} \cosh \lambda^{N b_{2}} e^{h}
$$

where $\lambda^{N b_{1}}, \lambda^{N b_{2}}$ and $h$ are evaluated with the particular value of $\left(\sigma^{F e_{1}}, \sigma^{F e_{2}}\right)$. Next step here would be to get eigenvalues of the $\mathbf{P}$ matrix, which in turn will give partition function for a particular orientation $(\theta, \phi, \psi)$ of the chain. Now Eq (16), Eq (17) and Eq (15) can be used for 


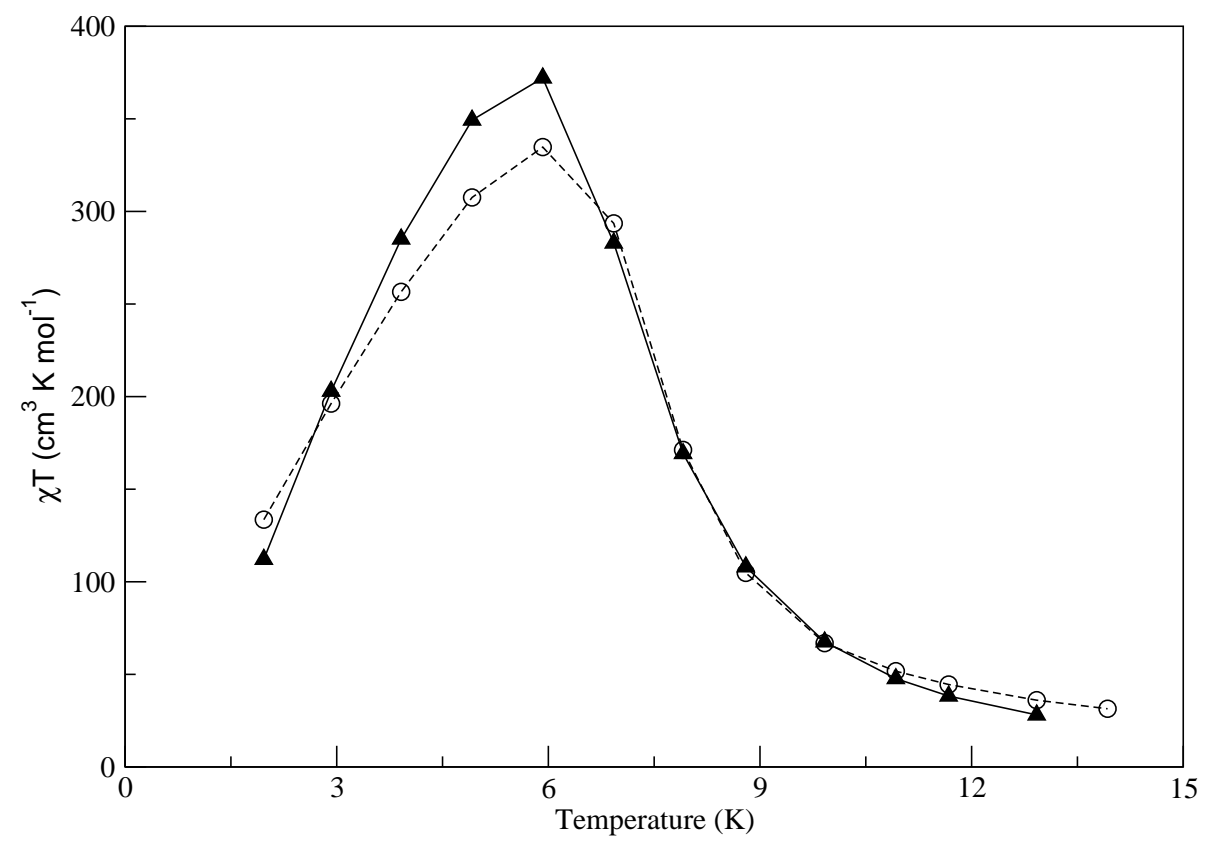

Figure 3: $\chi T v s . T$ curve for $\mathrm{Fe}_{2} \mathrm{Nb}$. Open circles $(\bigcirc)$, connected by segmented line, represent experimental data points whereas filled triangles $(\boldsymbol{\Lambda})$, connected by continuous line, represent calculated data points. The experiment was done with applied field of 100 Oe. All the theoretical points are calculated with same value of applied field.

obtaining $\chi T$ vs.T curve at low temperature (note, while Eq (17) will give $\chi_{0}$, Eq (15) will give us $\left.\chi_{M}\right)$.

\subsection{Result and Discussion}

In Fig (3) we show the best fit $\chi T v s . T$ plot with experimental data. The best fit parameters are: $J=20.3 \mathrm{~cm}^{-1}$ (antiferromagnetic), $N z J^{\prime}=2.3 \times 10^{-4} \mathrm{~cm}^{-1}$ (antiferromagnetic), $g=2.08$, $\alpha=33^{\circ}, \omega=22.5^{\circ}$ and $\delta=8^{\circ}$. The theoretical fit was obtained from 110 geometrical units or 220 chemical units. We observe that, below $6 \mathrm{~K}, \chi T$ value decrease with temperature due to two effects: (a) finite size of chains and (b) antiferromagnetic interchain interaction. Due to finite size, magnetization of the chain has an upper bound and $\chi$ reaches a saturation value and below this temperature, $\chi T$ value will begin to decrease. Effect of antiferromagnetic interchain interaction further reduces the value of susceptibility at low temperatures. We also note that, at higher temperatures theoretical value of susceptibility is lower compared to the experimental value. Since, unlike in a real system, the direction of $\mathrm{Fe}$ moments in the model are fixed and can not change, resulting in smaller magnetization than the powder compound. Two more things can be estimated from our model; first is the energy for creating a domain wall in the chain, $\Delta_{\xi}$, the second being the anisotropy constant, $D$.

One of the important properties of SCMs is the slow relaxation of magnetization. The 
relaxation dynamics is controlled by the blocking temperature of the system and is related to the energy required to create a domain wall in the chain. The lowest energy required to create a domain wall is the absolute difference in energy between the ground state and an exited state, in which one part of the chain is magnetized in one direction and the rest of the chain is magnetized in the opposite direction. In the class of SCMs we are discussing, visualization of domain wall may be difficult due to its complex structure, especially due to the presence of both quantum and classical units. It is sufficient here to consider each $\mathrm{Nb}$ ion and the associated off-chain $\mathrm{Fe}$ ion as one quantum unit, since all of these units are identical in the absence of external magnetic field. In order to get ground state energy of the SCM, we solve for the eigenvalues of a quantum unit for different configurations of the surrounding classical units. Here the Hamiltonian to be considered includes interaction of $\mathrm{Nb}$ ion with the associated off-chain $\mathrm{Fe}$ ion and half of the interactions of that $\mathrm{Nb}$ ion with surrounding in-chain $\mathrm{Fe}$ ions. We obtain the minimum energy when all the three Ising spins connected to the $N b$ ion are in same state, say, '+ 1 ' state. So the ground state of the chain is when all the Ising spins (both in- and off-chin) are in the ' +1 ' state. Now to create a domain wall, we reverse all the Ising spins of one part, say right half, and keep the remaining Ising spins of left half in the initial state of ' +1 '. This will change energy of only one quantum unit, where the off-chain and one in-chain Ising spins are in ' +1 ' state and another in-chain Ising spin is in '-1' state. The difference in energy, i.e., the energy to create a domain wall is given by (see Fig 1):

$$
\begin{aligned}
& \Delta_{\xi}=\frac{1}{2}\left(\left.\sqrt{a^{2}+b^{2}+c^{2}}\right|_{\sigma^{F e_{c}=1}}-\left.\sqrt{a^{2}+b^{2}+c^{2}}\right|_{\sigma^{F e_{c}=-1}}\right)_{\sigma^{F e_{1}=1, \sigma^{F e_{1}^{\prime}}}} \\
& \approx 34.5 \mathrm{~K}
\end{aligned}
$$

This compares well with the experimental energy gap of $33 \mathrm{~K}$ obtained from $\log (\chi T) \mathrm{vs} .1 / T$ plot above temperature of $6 \mathrm{~K}$ (to exclude effects due to finite size and interchain interactions).

Estimation of anisotropy parameter $(D)$ can not be done directly from the fits as our Hamiltonian does not contain the $D$ parameter. Here we employ an indirect way for its estimation. We have assumed that low temperature spins of $\mathrm{Fe}$-ions behave like Ising spins due to large and negative $D$-value from experiment. Spin of $\mathrm{Fe}$ ion being 2, we have also assumed that spin values that can be assumed by $F e$ is only \pm 2 . If we attribute the deviation of the theoretical curve from the experimental curve due to an assumption of canted Ising-type spin for the $\mathrm{Fe}$ spins, then from the deviation temperature $T_{d}$ (about $12 \mathrm{~K}$ in Fig 3 ) we can estimate the anisotropy parameter. To do this, we solve the quantum mechanical exchange Hamiltonian, namely, $H=J \overrightarrow{\mathbf{S}}^{F e} \cdot \overrightarrow{\mathbf{S}}^{N b}-D\left(\mathbf{S}_{Z}^{F e}\right)^{2}$, with both $J$ and $D$ positive, of 2-spin problem involving $F e$ and $\mathrm{Nb}$ spins exactly. Note, all the intrachain interactions are of this antiferromagnetic $\mathrm{Fe}-\mathrm{Nb}$ type. We conjecture that the deviation from the experimental $\chi T v s . T$ plot occurs due to significant population of the 3rd eigenstate relative to the second eigenstate. That is the thermal energy $k_{B} T_{d}$ is such that the ratio of the population of the 3rd eigenstate to the 2 nd eigenstate is $1 / e$. This yields the result that $\Delta E=E_{3}-E_{2} \approx 3 D-2 J=k_{B} T_{d}$. Since we know $J$ from the fitting, we estimate $D \sim 16.3 \mathrm{~cm}^{-1}$. This estimation is close to the experimental value of 17 $\mathrm{cm}^{-1}$. 


\section{Conclusion}

We have outlined a method which can be used to study thermodynamic properties of an important class of SCMs where alternate units are of classical and quantum nature with anisotropy axes of classical units being non-collinear. We have also discussed in detail the issue of interchain interaction and showed how to incorporate it in transfer matrix technique. We applied this method to a real system and studied its low temperature behavior. We have also carried out an averaging process that allows computing the magnetic properties of a powder sample. We have estimated anisotropy parameter and energy associated with domain wall within this model. At this point it is worth mentioning that, it may be better to consider all five states $(0, \pm 1, \pm 2)$ of the $z$-component of $\mathrm{Fe}$ spin instead of the two states considered here. However, in that case, one has to deal with little bigger transfer matrix (dimension $5 \times 5$ ); but the main disadvantage of this extension will be that it will add one more parameter to the Hamiltonian, as $D$ will now appear explicitly in the model.

\section{Acknowledgement}

We are thankful to the Department of Science and Technology (DST), India for financial support.

\section{References}

[1] Bogani, L., Vindigni, A., Sessoli, R., Gatteschi, D.: Single chain magnets: where to from here?. J. Mater. Chem. 18, 4750-4758 (2008)

[2] Miyasaka, H., Julve, M., Yamashita, M., Clérac, R.: Slow Dynamics of the Magnetization in One-Dimensional Coordination Polymers: Single-Chain Magnets. Inorg. Chem. 48, 34203437 (2009)

[3] Coulon, C., Clérac, R., Lecren, L., Wernsdorfer, W., Miyasaka, H.: Glauber dynamics in a single-chain magnet: From theory to real systems. Phys. Rev. B 69, 132408-132412 (2004)

[4] Vindigni, A.: Statistics and game theory in single-chain magnet relaxation. Inorg. Chim. Acta 361, 3731-3739 (2008)

[5] Sun, H.-L., Wang, Z.-M., Gao, S., Coord. Chem. Rev. 254, 1081-1100 (2010)

[6] Georges, R., Borrás-Almenar, J.J., Coronado, E., Curély, J., Drillon, M.: One-Dimensional Magnetism: An Overview of the Models. In: Miller, J.S., Drillon, M. (ed.) Magnetism: Molecules to Materials I, Wiley-VCH, New York ( 2001)

[7] Coulon, C., Miyasaka, H., Clérac, R.: Single-Chain Magnets: Theoretical Approach and Experimental Systems. Struct. Bond. 122 163-206 (2006) 
[8] Dupas, C., Renard, J.P., Seiden, J., Cheikh-Rouhou, A.: Static magnetic properties of $\left(\mathrm{CH}_{3}\right)_{4} \mathrm{NMn}_{x} \mathrm{Cu}_{1-x} \mathrm{Cl}_{3}$, a quantum ferromagnetic chain with classical impurities: Experiment and theory. Phys. Rev. B 25, 3261-3272 (1982)

[9] Seiden, J.: Propriétés statiques d'une chaîne isotrope alternée de spins quantiques $1 / 2$ et de spins classiques. J. Physique Lett. 44, 947-952 (1983)

[10] Verdaguer, M., Gleizes, A., Renard, J.P., Seiden, J.: Susceptibility and magnetization of $\mathrm{CuMn}\left(\mathrm{S}_{2} \mathrm{C}_{2} \mathrm{O}_{2}\right)_{2} \cdot 7.5 \mathrm{H}_{2} \mathrm{O}$. First experimental and theoretical characterization of a quasi-onedimensional ferrimagnetic chain. Phys. Rev. B 29, 5144-5155 (1984)

[11] Georges, R., Curély, J., Gianduzzo, J.C., Qiang, Xu, Pei, Y.: Alternating quantumclassical spin Heisenberg chains: Exchange and /or cation random distribution effects. Physica B 153, 77-84 (1988)

[12] Curély, J., Georges, R., Drillon, M.: Local-anisotropy and spin-nature effects in ferrimagnetic Ising chains: New compensation phenomena. Phys. Rev. B 33, 6243-6252 (1986)

[13] Pełka, R., Bałanda, M., Przychodzeń, P., Tomala, K., Sieklucka, B., Wasiutyński, T.: Generalized theoretical approach to quasi-one-dimensional molecular magnets. phys. stat. sol. (c) 3, 216-219 (2006)

[14] Strečka, J., Michal Jaščur, M.: Existence of a magnetization plateau in a class of exactly solvable IsingHeisenberg chains. J. Phys.: Condens. Matter 1545194534 (2003)

[15] Chandra, V.R., Ramasesha, S., Sen, D.: Magnetic properties of a helical spin chain with alternating isotropic and anisotropic spins:Magnetization plateaus and finite entropy. Phys. Rev. B 70, 144404-144412 (2004)

[16] Venkatakrishnan, T.S., Sahoo, S., Bréfuel, N., Duhayon, C., Paulsen, C., Barra, A.-L., Ramasesha, S., Sutter, J.-P.: Enhanced Ion Anisotropy by Nonconventional Coordination Geometry: Single-Chain Magnet Behavior for a $\left[\left\{\mathrm{Fe}^{I I} \mathrm{~L}\right\}_{2}\left\{\mathrm{Nb}^{I V}(\mathrm{CN})_{8}\right\}\right]$ Helical Chain Compound Designed with Heptacoordinate $\mathrm{Fe}^{I I}$. J. Am. Chem. Soc. 132, 6047-6056 (2010)

[17] Ferbinteanu, M., Miyasaka, H., Wernsdorfer, W., Nakata, K., Sugiura, K., Yamashita, M., Coulon, C., Clérac, R.: Single-Chain Magnet $\left(\mathrm{NEt}_{4}\right)\left[\mathrm{Mn}_{2}(5-\mathrm{MeOsalen})_{2} \mathrm{Fe}(\mathrm{CN})_{6}\right]$ Made of $\mathrm{Mn}^{I I I} \mathrm{Fe}^{I I I} \mathrm{Mn}^{I I I}$ Trinuclear Single-Molecule Magnet with an $\mathrm{S}_{T}=9 / 2$ Spin Ground State. J. Am. Chem. Soc. 127, 3090-3099 (2005)

[18] Clérac, R., Miyasaka, H., Yamashita, M., Coulon, C.: Evidence for Single-Chain Magnet Behavior in a $\mathrm{Mn}^{I} I I \mathrm{Ni}^{I} I$ Chain Designed with High Spin Magnetic Units: A Route to High Temperature Metastable Magnets. J. Am. Chem. Soc. 124, 12837-12844 (2002)

[19] Zheng, Y.-Z., Tong, M.-L., Zhang, W.-X., Chen, X.-M.: Assembling Magnetic Nanowires into Networks: A Layered $\mathrm{Co}^{I I}$ Carboxylate Coordination Polymer Exhibiting Single-ChainMagnet Behavior. Angew. Chem. Int. Ed. 45, 6310-6324 (2006) 